\title{
Parentalitat i parla adreçada als infants: com els adults promouen el desenvolupament comunicatiu i lingüístic infantil
}

\section{Magdalena Rivero García i Rosa Vilaseca Momplet}

Departament de Cognició, Desenvolupament i Psicologia de l'Educació, Facultat de Psicologia, Universitat de Barcelona.

mriverog@ub.edu

rosavilaseca@ub.edu

Recepció: 27/03/2019, acceptació: 28/06/2019

Resum: En aquest article oferim una revisió actualitzada dels principals resultats de les investigacions sobre el desenvolupament inicial de la comunicació i del llenguatge, posant l'èmfasi en com els pares i les mares, $i$ en general els adults significatius per als nens $\mathrm{i}$ les nens, promouen el desenvolupament de les habilitats comunicatives i lingüístiques infantils.

Inicialment, revisem les característiques dels primers intercanvis comunicatius entre bebès i adults, centrant-nos especialment en com evolucionen l'atenció conjunta i la intencionalitat en el marc d'aquests intercanvis. Posteriorment, abordem com determinats comportaments parentals que defineixen la parentalitat positiva s'han mostrat relacionats amb el desenvolupament infantil en general i amb el desenvolupament comunicatiu i lingüístic en particular. També ens referim a les característiques de la parla adreçada als infants que, d'acord, amb les investigacions, donen suport al procés d'adquisició del llenguatge. Acabem senyalant algunes implicacions pràctiques dels resultats de les investigacions, a prendre en consideració en la intervenció professional amb les famílies i els educadors dels nens i les nenes que requereixen una atenció especial en l'àrea de la comunicació i del llenguatge.

Paraules clau: parentalitat positiva, responsivitat parental, parla adreçada als infants, desenvolupament comunicatiu i lingüístic, intervenció precoç i naturalista, Enfocament Centrat en la Família.

\section{Parentalidad y habla dirigida al niño: cómo los adultos promueven el desarrollo comunicativo y lingüístico infantil}

Resumen: En este artículo ofrecemos una revisión actualizada de los principales resultados de las investigaciones sobre el desarrollo inicial de la comunicación y del lenguaje, poniendo el énfasis en como los padres y las madres, y en general los adultos significativos para los niños y las niñas, promueven el desarrollo de las habilidades comunicativas y lingüísticas infantiles.

Inicialmente, revisamos las características de los primeros intercambios comunicativos entre bebés y adultos, centrándonos especialmente en cómo evolucionan la atención conjunta y la intencionalidad en el marco de esos intercambios. Posteriormente, abordamos cómo determinados comportamientos parentales que definen la parentalidad 
positiva se han mostrado relacionados con el desarrollo infantil en general y con el desarrollo comunicativo y lingüístico en particular. También nos referimos a las características del habla dirigida a los niños que, de acuerdo con las investigaciones, apoyan el proceso de adquisición del lenguaje. Finalizamos señalando algunas implicaciones prácticas de los resultados de las investigaciones, a tomar en consideración en la intervención profesional con las familias y los educadores de los niños y niñas que requieren una atención especial en el área de la comunicación y del lenguaje.

Palabras clave: parentalidad positiva, responsividad parental, habla dirigida al niño, desarrollo comunicativo y lingüístico, intervención precoz y naturalista, modelo centrado en la familia.

\section{Parenting and Child Directed Speech: How Do Adults Promote the Communicative and Linguistic Development}

Abstract: In this paper we offer an updated review of the main results of research on early communicative and linguistic development, emphasizing how fathers and mothers, and in general, significant adults for children, promote the development of children's communicative and linguistic skills.

Initially, we review the characteristics of the first communicative exchanges between babies and adults, focusing especially on how joint attention and intentionality evolve within the framework of these exchanges. Subsequently, we address how certain parental behaviors that define positive parenting have been shown to be related to child development in general and to communicative and linguistic development in particular. We also refer to those characteristics of child directed speech that, according to research, support the process of language acquisition. We conclude by pointing out some practical implications of the results of the research, to be considered in professional intervention with the families and educators of children who require special attention in the area of communication and language.

Keywords: positive parenting, parental responsivity, Child Directed Speech, communicative and linguistic development, naturalistic and early intervention, Family Centered Approach.

\section{INTRODUCCIÓ}

L'objectiu principal d'aquest treball és donar a conèixer els resultats dels estudis sobre el desenvolupament inicial de la comunicació i del llenguatge, tant propis com d'altres investigadors, centrant-nos en com els pares i les mares, i en general els adults significatius per als nens i les nenes, promouen, de manera sovint més inconscient que voluntària, el desenvolupament de les habilitats comunicatives i lingüístiques infantils. Els destinataris de les idees que exposarem a continuació són les famílies, els educadors infantils $\mathrm{i}$ tots aquells professionals que, en contextos diversos (Centres de Desenvolupament Infantil i Atenció Precoç, escoles bressol, espais familiars, escoles d'educació especial, programes d'educació parental, serveis socials...), treballen conjuntament amb les famílies per millorar la qualitat dels processos interactius entre adults i infants, amb l'objectiu final de promoure el bon desenvolupament comunicatiu i lingüístic dels nens i les nenes. 
Cercant la claredat, organitzarem l'exposició en diversos apartats, amb el benentès que els diferents aspectes tractats estan interrelacionats i que en el dia a dia de les famílies es concreten d'una manera integrada i incardinada en activitats de la vida quotidiana, com són, per exemple, l'alimentació, el canvi de bolquers, el joc durant el bany o les sortides al parc. Aquestes i altres situacions de la vida diària són el rerefons del que exposarem a continuació.

\section{LES PRIMERES INTERACCIONS ENTRE ELS BEBÈS I ELS}

\section{ADULTS}

Avui dia sabem que els bebès humans neixen amb habilitats perceptives $\mathrm{i}$ de processament de la informació especialment adaptades per analitzar els estímuls que provenen dels humans. Els nounats se senten especialment atrets pels rostres (Slater i Quinn 2001); durant els primers dies de vida poden mantenir el contacte visual amb l'adult durant alguns segons i tendeixen a reproduir moviments facials realitzats per un adult davant seu, com ara l'obertura de la boca o la protrusió de la llengua (Meltzoff i Moore 1997), i esperen que hi hagi sincronia entre l'expressió facial i la veu de les persones (Murray i Trevarthen 1985; Tronick, Als i Adamson 1979).

Els nounats també responen amb interès a la veu humana (Vouloumanos i Werker 2007); poden identificar els fonemes de totes les llengües del món i diferencien els idiomes, tot i que prefereixen escoltar els del seu entorn (Mehler, Betoncini, Barrière i Jassik-Gerchenfeld 1978; Moon, Cooper i Fifer 1993). És interessant el fet que al llarg dels primers mesos de vida es produeixen processos de reorganització perceptiva que porten a una pèrdua d'aquesta capacitat inicial de diferenciar fonemes de totes les llengües i a una especialització en la distinció dels fonemes de les llengües a les quals el bebè està exposat. Aquesta reorganització té lloc cap als sis mesos per a les vocals i entre els deu i dotze mesos per a les consonants (Bosch i Sebastián-Gallés 2003; per a una revisió recent vegeu Rivero 2018). Els nounats també mostren preferència per les unitats de parla que s'aproximen a la sílllaba i per les cadenes de parla que tenen una durada similar a la longitud aproximada d'una frase (Aslin, Pisoni i Jusczyk 1983; Hirsh-Pasek et al. 1987).

Des dels primers dies de vida els bebès comencen a establir relacions amb els seus cuidadors. Els comportaments del nadó i els de l'adult s'alternen, es coordinen i se sincronitzen, de manera que adult i bebè arriben a establir autèntics processos d'interacció amb ajustaments mutus. El bebè para atenció al rostre de l'adult quan aquest el mira, reacciona als seus estats emocionals i tendeix a sincronitzar els seus moviments amb la veu de l'adult quan aquest li parla. Durant la interacció, l'adult intenta captar l'atenció del bebè i fa pauses esperant la seva resposta. Els pares i les mares tendeixen a encavalcar les seves vocalitzacions amb les del bebè per intentar mantenir-les i allargarles quan es produeixen. El bebè, al seu torn, respon a l'adult de diverses maneres, com ara amb moviments corporals o dels llavis, les expressions facials o les vocalitzacions.

Aquests primers intercanvis entre bebès $\mathrm{i}$ adults, en els quals les característiques principals són la sincronia, l'ajustament mutu, la reciprocitat i l'alternança, han estat denominats protoconverses, donat que constitueixen autèntics processos interactius 0 «diàlegs». Ja fa algunes dècades, Trevarthen (1980) va proposar el terme intersubjectivitat primària per referir-se a la motivació especial que senten els bebès per a les interaccions amb les persones i per al conjunt d'habilitats que fan possible que, des dels primers dies de vida, puguin mantenir-les. D'altra banda, resulta obvi que les competències comunicatives d'adults i infants són molt diferents. Els bebès encara no dominen els codis de la comunicació humana i el llenguatge, però l'adult sovint interpreta 
les accions del bebè com autèntics intents de comunicació i de parla, atribuint-los significats específics com ara «Dius que et doni ja el menjar, oi?», «Tens son» o «Vols jugar amb el papa». Autors com Kaye (1982) han destacat que els pares i les mares, tot atribuint significat a les accions, les expressions o les vocalitzacions del bebè, el van introduint en els marcs socials i culturals en què tindrà lloc l'adquisició del llenguatge.

Cap al tercer o quart mes de vida, augmenta de manera exponencial l'interès del bebè pels objectes del món que l'envolta. Aquest interès està relacionat amb la millora de la visió i de les destreses motores. L'adult és sensible als nous interessos de l'infant. Quan detecta que la mirada del bebè s'adreça, per exemple, a una joguina, tendirà a acostar-sela, a dir frases com "Vols la piloteta?", "Sí, és una pilota" o "T'agrada la pilota", o a fer alguna acció interessant amb la pilota per mantenir l'interès del bebè o per augmentar-lo amb algun efecte sorpresa, com ara llençar la pilota al terra tot dient "pumba!".

A partir dels quatre mesos aproximadament els bebès es van incorporant progressivament a aquesta mena de jocs senzills amb l'adult (Bruner 1983). De manera natural, l'adult intenta que el bebè participi en el joc, ajustant les seves demandes a allò que el bebè és capaç de fer en cada moment. Així, per exemple, l'adult pot fer una torre de cubs i animar el bebè perquè li faci un cop amb la mà per derrocar-la. L'adult donarà temps al bebè perquè faci la seva part en el joc i li proporcionarà ajuda si cal, per exemple, guiant-li la mà cap a la torre. Altres jocs senzills en els quals sovint participen el bebè $\mathrm{i}$ l'adult són els de les cançonetes infantils associades a moviments de les mans (per exemple, «ralet-ralet, pica-pica dineret», «en Jan petit quan balla»...), el joc del cucut-tat (amagar el rostre darrere de les mans o d'una tovallola i reaparèixer) o activitats com ara llençar i agafar del terra un objecte de manera reiterada, picar de mans, donar copets rítmics a la taula i molts d'altres. Sovint les paraules acompanyen accions o esdeveniments rellevants del joc (per exemple, «pum» o «ooooh!» quan l'objecte cau, «plis plas» quan es pica de mans...). Tots aquests jocs tenen en comú que adult i bebè hi participen activament, fent cadascun d'ells el seu rol, de manera alterna. Inicialment, el rol del bebè serà sempre el més simple dels que conformen el joc (qui derroca la torre, qui llença l'objecte, qui mou la mà mentre la mare canta «En Jan petit»...). Però a mida que el nen domini el joc i augmentin les seves destreses podrà desenvolupar qualsevol dels rols del joc, esdevenint aquests intercanviables.

Bruner (1983) va estudiar amb molt detall aquests jocs d'intercanvi i els va relacionar amb el desenvolupament de la comunicació i del llenguatge. D'una banda, aquests jocs permeten al bebè anar dominant les normes bàsiques de tota conversa: l'alternança de torns (ara intervé un participant, després l'altre) i l'alternança de rols (cada participant agafa el rol de parlant i d'oient de manera alterna). D'altra banda, els jocs d'intercanvi que formen part de la vida quotidiana del bebè es repeteixen una vegada i una altra, amb petites variacions; tenen una seqüència clara d'accions i paraules que el bebè pot arribar a predir, i les accions i les paraules prenen significat per a l'infant en el context del joc. És per tot això que aquests jocs constitueixen contextos idonis per al desenvolupament de la comunicació i del llenguatge.

Els bebès de cinc $\mathrm{o}$ sis mesos d'edat segueixen la mirada de l'adult quan aquest para atenció a algun objecte, persona o esdeveniment de l'entorn. I tornen a inspeccionar la mirada de l'adult si no aconsegueixen identificar què està mirant. Durant la segona meitat del primer any de vida la capacitat del bebè per mantenir l'atenció sobre alguna cosa al mateix temps que ho fa l'adult es desenvolupa molt significativament. És el que es coneix com a desenvolupament de les habilitats d'atenció conjunta (Tomasello i Farrar 1986; Rivero 2006, 2018; Prieto et al. 2015). Cap als nou mesos d'edat, els bebès identifiquen amb facilitat què està assenyalant un adult, sempre que l'objecte, persona o esdeveniment 
assenyalat estigui a prop i sigui clarament identificable (Leung i Rheingold 1981). Els adults generalment intenten facilitar al bebè la tasca d'identificació, mirant alternativament cap al rostre del bebè i cap allò que s'assenyala -generalment un objecte-, fent moviments emfàtics amb el cap, insistint en el manteniment de la mirada cap a l'objecte i assegurant-se que aquest es troba dins del camp visual del bebè (Rivero 2018).

Així mateix, a partir dels nou o deu mesos d'edat, quan el bebè vol que l'adult es fixi en alguna cosa que a ell l'interessa és capaç de fer-ho entendre, coordinant accions dirigides a l'objecte i accions dirigides a l'adult. Així, per exemple, el bebè mira alternativament cap allò que l'interessa i cap a l'adult, tot vocalitzant i estenent la mà cap a l'objecte d'interès. Aquesta capacitat per integrar el món de les coses amb el món de les persones és el que Trevarthen $(1980,2015)$ va denominar intersubjetivitat secundària. La intersubjectivitat secundària coincideix amb la realització, per part del bebè, d'accions intencionals (Piaget 1936) i amb la capacitat per interpretar com intencionals les accions dels altres (Vouloumanos, Onishi i Pogue 2012). S'entén com a conducta intencional aquella que coordina una meta o finalitat amb uns mitjans per aconseguir-la. En el context de la comunicació, el bebè disposa ja de les habilitats necessàries per interpretar les intencions dels adults i per expressar ell mateix les seves pròpies intencions comunicatives (Tomasello 1999). Diversos estudis fets amb bebès (per exemple, Ninio 1993; Snow, Pan, Imbens-Bailey i Herman 1996; Rivero 2006) identifiquen el ventall d'intencions comunicatives que els bebès expressen abans de parlar, com per exemple, demanar un objecte, expressar el desig d'iniciar una activitat, expressar el desig de continuar l'activitat en curs, demanar ajuda o expressar malestar.

Convé remarcar que les habilitats d'atenció conjunta, que es van desenvolupant progressivament al llarg del primer any de vida i que es consoliden en el darrer trimestre d'aquest primer any són cabdals per al desenvolupament comunicatiu i lingüístic posterior. Alguns estudis han mostrat que la quantitat de temps que els bebès participen en interaccions d'atenció conjunta amb els adults (per exemple, apilant blocs, jugant a titelles o mirant contes) és un bon predictor del desenvolupament lingüístic posterior (Carpenter, Nagel i Tomasello 1998).

\section{Parentalitat Positiva: interaccions Parentals}

\section{AFAVORIDORES DEL DESENVOLUPAMENT DEL LLENGUATGE}

Una funció cabdal de la família és allò que comunament es coneix com a criança dels fills. Són moltes les investigacions que actualment es plantegen identificar els trets definitoris del que seria una bona criança, és a dir, una criança realment afavoridora del benestar, la salut i el desenvolupament de l'infant, que alhora promogui i preservi el benestar de la família en el seu conjunt. Aquests estudis empren sovint el terme parentalitat o l'expressió parentalitat positiva per referir-se a la cura, l'atenció, l'educació i la socialització dels fills i filles. Dins del conjunt d'activitats, situacions i processos que engloba l'ampli concepte de parentalitat tenen un especial interès en relació amb el desenvolupament del llenguatge, les interaccions que mares i pares mantenen amb els seus fills i filles en les activitats de la vida diària, a les quals ens referim com a interaccions parentals.

En els darrers anys són moltes les investigacions que han posat el focus a identificar quines característiques de les interaccions parentals resulten més facilitadores o optimitzadores del desenvolupament infantil. Per a l'estudi d'aquestes característiques s'ha recorregut principalment als qüestionaris per als pares o a l'observació directa de les 
interaccions entre pares i fills. Un instrument observacional que actualment s'està emprant en diversos països per a l'anàlisi de les interaccions parentals amb nens d'entre 10 i 47 mesos d'edat és PICCOLO (Parenting Interactions with Children: Checklist of Observations Linked to Outcomes; Interaccions parentals amb els infants: inventari d'observacions lligades a resultats; Roggman, Cook, Innocenti, Jump Norman i Christiansen 2013). Aquest instrument, que ha estat validat en població espanyola pel nostre grup d'investigació (Vilaseca et al. 2019), recull un total de 29 comportaments parentals que, després d'una àmplia revisió dels estudis previs i de les pròpies investigacions, els autors originals van identificar com a clarament relacionats amb un bon desenvolupament infantil.

Els 29 comportaments parentals o ítems de PICCOLO estan agrupats en quatre subescales: afecte (Affection), responsivitat (Responsiveness), encoratjament (Encouragement) i ensenyança (Teaching). Seguidament ens referirem a cadascuna d'aquestes quatre dimensions.

L'afecte es manifesta a través de comportaments com el somriure, la calidesa de la veu, la proximitat física durant el joc o l'elogi de les accions positives del nen. L'afecte o calidesa emocional dels pares i mares envers els seus fills s'ha relacionat amb el desenvolupament socioemocional de l'infant i amb la formació d'un vincle afectiu sa, basat en la seguretat i la confiança. No disposem d'estudis que relacionin específicament l'afecte parental i el desenvolupament de la comunicació i del llenguatge. No obstant això, la influència seria indirecta, a través de la qualitat del vincle i de l'estat emocional de l'infant, ja que un bon vincle amb els cuidadors principals promou el desenvolupament general de l'infant (Kochanska 2001; Zhou et al. 2002).

La responsivitat és una de les característiques de les interaccions parentals que més clarament s'ha relacionat amb el desenvolupament de la comunicació i del llenguatge (Goldstein i Schwade 2008; Nicely, Tamis-LeMonda i Bornstein 1999). Diem que un adult és responsiu quan està atent als senyals que dona l'infant sobre el seu estat emocional i els seus interessos i respon a aquests senyals de manera ràpida i ajustada (Tamis-LeMonda, Kuchirko i Song 2014). La responsivitat s'observa en comportaments parentals com estar atent a allò que l'infant fa o intenta fer, ajustar el ritme de l'activitat a les necessitats del nen, respondre als seus intents de comunicació o reaccionar a les seves emocions. Per exemple, un pare o una mare està mirant un conte amb la seva filla i aquesta dona senyals d'avorriment $o$ desinterès. Possibles respostes responsives per part de l'adult serien preguntar a la nena si està cansada del conte, si en vol mirar un altre, preguntar-li què vol fer... Pel contrari, la insistència en la lectura del mateix conte malgrat el desinterès de l'infant seria una resposta no responsiva per part de l'adult, que en aquest cas es mostraria directiu.

Tamis-LeMonda et al. (2014) senyalen que les respostes adultes responsives afavoreixen el desenvolupament comunicatiu i lingüístic per diverses raons. D'una banda, les respostes ràpides $\mathrm{i}$ connectades amb allò que el nen està fent $\mathrm{o} a \mathrm{mb}$ allò en què centra l'atenció (respostes contingents) afavoreix que el nen estableixi relacions entre els objectes, les accions o esdeveniments i les expressions verbals i no verbals. Un estudi recent de McGillion, Pine, Herbert i Matthews (2017) mostra la importància de la parla adulta contingent per a l'adquisició de vocabulari durant el segon any de vida i presenta els resultats d'un programa d'intervenció amb famílies d'estatus socioeconòmic baix. D'altra banda, les respostes adultes en forma de preguntes i denominacions o descripcions dels objectes, promouen la participació del nen en la conversa i aporten models lingüístics a l'infant. A més, el fet que els adults coordinin generalment comportaments no verbals (mirar, tocar, assenyalar, oferir...) i verbals (paraules o frases 
curtes) afavoreix la comprensió i l'aprenentatge del llenguatge.

Un altre grup de comportaments parentals relacionats amb el desenvolupament infantil són aquells que tenen a veure amb la promoció de l'autonomia, l'establiment de límits i les exigències de maduresa, sempre d'acord amb el nivell evolutiu de l'infant. Corresponen a la dimensió que a PICCOLO es denomina encoratjament i es manifesten quan la mare o el pare animen l'infant a prendre la iniciativa durant el joc, l'encoratgen a fer les coses per si mateix, mostren entusiasme quan el nen està fent quelcom que li suposa un esforç o li fan suggeriments sense interferir en el seu joc. La directivitat intrusiva seria el contrari de l'encoratjament. Per exemple, una mare i un nen estan resolent un puzle. El nen no en domina la resolució a la perfecció i ha d'anar a poc a poc, mirant les peces amb atenció per seleccionar-ne l'adequada. A més, quan la troba, li costa encaixar-la al seu lloc. Una mare que promou l'autonomia l'animarà a buscar les peces, li donarà pistes, esperarà la resposta de l'infant després de fer-li un suggeriment i li oferirà l'ajuda indispensable per avançar en la tasca, intentant que el nen faci tot allò que pugui fer per si mateix. Contràriament, una mare directiva podria mostrar comportaments com treure al nen la peça de la mà quan li costa encaixar-la per posar-la-hi ràpidament ella, fer-li preguntes i respondre-les ella mateixa tot seguit o indicar al nen quina peça agafar sense donar-li temps a pensar.

La directivitat intrusiva de les mares, amb qui s'ha fet la major part de les investigacions, s'ha associat a retards en el desenvolupament de la comunicació per part del nen (Girolametto i Tannock 1994). Pel contrari, l'encoratjament s'ha relacionat positivament amb el desenvolupament inicial del llenguatge (Cielinski et al. 1995; Roach, Barratt, Miller i Leavitt 1998).

Finalment, pel que fa a les característiques generals de les interaccions parentals que promouen el desenvolupament infantil, hem d'assenyalar la importància de l'estimulació cognitiva i lingüística, identificada a PICCOLO com a ensenyança, que inclou, per exemple, explicar a l'infant el perquè de les coses, fer-li preguntes, donar nom als objectes o parlar de les seves propietats (color, mida, forma...). Les investigacions han relacionat clarament aquests comportaments parentals amb el desenvolupament del llenguatge (Tamis-LeMonda, Bornstein i Baumwell 2001; Hubbs-Tait, Culp, Culp i Miller 2002; Kim-Cohen, Moffitt, Caspi i Taylor 2004; Bingham 2007).

Per concloure aquest apartat, volem assenyalar que els nostres propis estudis, actualment en curs i fets amb famílies amb infants amb desenvolupament normatiu i famílies amb infants amb discapacitat intel-lectual (DI), també indiquen una relació positiva entre les interaccions parentals de qualitat i el desenvolupament del llenguatge. D'acord amb els resultats que fins ara hem analitzat, que corresponen als infants amb DI, les interaccions responsives i l'estimulació cognitiva i lingüística, tal com les hem descrit anteriorment, afavoririen el desenvolupament lingüístic inicial (Vilaseca et al. 2018).

En relació amb aquest punt, també volem destacar que, tot i que la major part de les investigacions sobre parentalitat han estat fetes amb mares, en els darrers anys han augmentat les relatives als pares (Cabrera et al. 2011; Kochanska i Kim 2013; Lamb 2010; Meuwissen i Carlson 2015). Els nostres estudis, que inclouen pares i mares, mostren que, tant els uns com les altres disposen de les competències necessàries per promoure el desenvolupament infantil a través d'interaccions positives amb els seus fills i filles (Rivero et al. 2019). 


\section{PARLA ADREÇADA A L'INFANT}

Un aspecte específic de les interaccions entre adults i infants és la parla adreçada als nens en el context de les interaccions. És el que es coneix com a parla d'estil matern o parla materna (motherese) o, més recentment, com a parla adreçada a l'infant (Infant or Child Directed Speech-CDS). L'estudi d'aquesta parla és especialment rellevant per entendre com els adults afavoreixen en el desenvolupament comunicatiu i lingüístic infantil.

Des dels anys cinquanta del segle vint s'han analitzat les característiques que diferencien la parla que els adults adrecen als nens petits de la parla pròpia de les converses entre adults (per a una revisió vegeu, per exemple, Rivero 1993; Serra, Serrat, Solé, Bel i Aparici 2000). Aquestes característiques s'han identificat en la parla de les mares i dels pares, en la de persones sense fills i/o amb poca experiència de tracte amb infants, i en la dels nens de quatre anys endavant quan s'adrecen a nens més petits. S'han constatat en una gamma molt àmplia de llengües, sense arribar a ser totalment universals, ja que alguns grups culturals organitzen de manera diferent els intercanvis comunicatius entre adults i infants. No obstant això, aquestes adaptacions de la parla són la norma en el nostre context sociocultural quan ens adrecem als nens que estan en les etapes inicials de l'adquisició del llenguatge (Ochs i Schieffelin 1995). D'altra banda, cal entendre aquestes adaptacions com a quelcom dinàmic, que canvia en funció de l'edat $\mathrm{i}$ del nivell de desenvolupament lingüístic de l’infant (Snow 1995).

Un dels trets més evidents de la parla adreçada a l'infant - a partir d'ara CDS-, per comparació amb la parla entre adults, és el to de veu elevat, l'exageració dels contorns de l'entonació (de vegades fins i tot pot semblar una cantarella) i l'alentiment del ritme (Broen 1972; Garnica 1977; Sachs 1977), així com l'allargament de les síllabes, especialment de les vocals (Burnham, Kitamura i Vollmer-Conna 2002; Liu, Kuhl i Tsao 2003). A la CDS també es tendeix a separar els enunciats per pauses relativament llargues. Aquesta parla resulta especialment atractiva per als infants, així com més clara.

La simplicitat dels enunciats, des d'un punt de vista gramatical, és una altra característica de la CDS. Parlem als infants amb enunciats curts i senzills des del punt de vista gramatical. En aquest sentit, Brent i Siskind (2001) van mostrar que la major exposició dels bebès a enunciats d'una sola paraula augmentava la probabilitat que ells diguessin aquella paraula entre l'any i l'any i mig d'edat.

La redundància o repetició és un altre tret propi de la CDS (Snow 1977). Els nadons se senten especialment atrets per la reiteració (McRoberts, McDonough, i Lakusta 2009) i aquesta facilitaria l'adquisició del llenguatge (Newman, Rowe, i Bernstein Ratner 2015).

Quant al vocabulari i al contingut del llenguatge - de què parlem als nens petits-, s'ha observat que inicialment predomina la parla referida a objectes, persones, accions i esdeveniments presents i observables en el context immediat (Tomasello i Farrar 1986). A mida que l'infant es desenvolupa cognitivament i lingüísticament, els adults van introduint referències a elements no presents, passats o futurs, explicacions del perquè de les coses i parla en el context de la ficció i del simbolisme (per exemple, dir coses posant veu de personatge malvat o parlar en nom de l'animal d'un conte) (Rowe 2012).

A més d'adaptar la parla al nivell evolutiu de l'infant, els adults intenten promoure que ell participi activament a la conversa. Un objectiu important per a l'adult és que el nen prengui el seu torn (Snow 1977), cercant un equilibri entre els torns assumits per l'adult i els protagonitzats per l’infant (Rivero, Fernández-Viader i Gràcia 1995). A més, els adults prioritzen l'expressió del conjunt d'intencions comunicatives que els infants poden comprendre (Pan, Imbens-Beiley, Winner i Snow 1996). Així, per exemple, negociar 
l'activitat conjunta, fer comentaris sobre el focus de l'atenció compartida i assenyalar esdeveniments específics, com ara la desaparició d'un objecte, serien els propòsits comunicatius més freqüents entre la mare i l'infant durant el primer any de vida (Rivero 2010).

Molt rellevants, i molt relacionades amb l'estimulació cognitiva i lingüística a què ens hem referit amb anterioritat, són les estratègies que empra l'adult per establir lligams o vinculacions entre la seva pròpia parla i la parla de l'infant (Rivero 1993). Així, entre aquestes estratègies, podem identificar les «imitacions parcials o idèntiques de la parla infantil» (per exemple, Nen: Mama. Mare: Mama.); les «correccions implícites» o correccions sense retroalimentació negativa, és a dir, sense dir al nen explícitament que ha pronunciat una paraula de manera diferent de com es pronunciaria (Nena: Totxe. Pare: Cotxe.); les «extensions» o ampliacions de l'enunciat fent-lo més complert des del punt de vista gramatical (Nen: Nena. Pare: És una nena.); les «extensions semàntiques» 0 ampliacions de l'enunciat introduint algun matís nou de significat (Nena: Nen. Mare: El nen és maco.) o la «descontextualització», que consisteix a relacionar el que acaba de dir l'infant amb alguna experiència del passat o del futur (Nen: A gat e banc. Mare: Sí, un gat blanc com el que vam veure a casa de l'àvia.).

Existeixen nombroses investigacions que vinculen les característiques de la CDS amb el desenvolupament lingüístic i comunicatiu (Hoff 2006; Rivero, Vilaseca i Borneis 2017; Rowe 2012). No obstant això, encara queda camí per recórrer en l'estudi d'aquesta temàtica, precisant millor les relacions entre aspectes específics de la CDS i el llenguatge infantil, i estudiant les relacions de la CDS amb trets generals de la parentalitat, les diferències en funció del context $o$ situació, $o$ les similituds, diferències i complementarietats entre progenitors.

\section{DE LA investigació A la intervenció: intervenció NATURALISTA SOCIOPRAGMÀTICA}

Tot el que s'ha anat comentant fins ara avala la naturalesa social, pragmàtica i cultural del desenvolupament humà, tant en infants de desenvolupament típic com amb discapacitat $\mathrm{o} a \mathrm{mb}$ risc de presentar algun retard en el desenvolupament. Reconèixer que el tipus d'interacció social i lingüística que mantenen les mares i pares amb els seus fills i filles a través de les rutines diàries pot influir en els processos d'adquisició i desenvolupament del llenguatge, té una forta implicació per a la intervenció amb aquelles famílies que tenen infants amb retard en aquesta àrea $\mathrm{o} a m b$ risc de tenir-ne.

Aquesta idea no és nova. A finals de la dècada de 1970, van començar a aparèixer tècniques d'intervenció anomenades naturalistes, basades en les teories interactives i sociofuncionals que fonamenten moltes de les investigacions esmentades en els apartats anteriors. Segons Del Rio (2006), la intervenció naturalista consisteix en un conjunt de procediments psicoeducatius destinats a facilitar el desenvolupament de la comunicació i del llenguatge, basant-se en adaptacions dels suports i de les estratègies naturals que pares i mares, i adults en general, ofereixen als infants en situacions de la vida quotidiana per optimitzar el desenvolupament en aquest àmbit. Els pares recorren a aquests suports de manera intensiva, no planificada i no intencional. La reconversió d'aquests suports en estratègies d'intervenció intencionada és el nucli de la intervenció naturalista. Molts van ser els treballs de recerca que van dur a terme una intervenció naturalista per facilitar el desenvolupament del llenguatge i la comunicació amb infants amb discapacitat intel-lectual (DI) (Vilaseca 2002), amb famílies que tenen fills amb DI (Gràcia 2000) o en el context escolar amb infants amb necessitats educatives especials (Sánchez-Cano 2001; 
Urquía 1998). No obstant això, eren programes d'intervenció o bé centrats en el nen o bé centrats en els cuidadors, basats en el model expert. Era el psicòleg qui, seguint els models sociofuncionals, donava pautes de com interactuar d'una manera eficaç amb l'infant amb retard en l'àrea del llenguatge i la comunicació. L'aposta per una intervenció en el context natural ha ressorgit amb força en l'àmbit internacional quan els programes d'intervenció es basen en la parentalitat o en les interaccions recíproques que estableixen els adults amb els seus fills en el seu context natural seguint un enfocament centrat en la família (ECF) (Dunst i Espe-Sherwindt 2016). Aquestes propostes han tingut un impacte molt important en la intervenció en l'àrea de la comunicació i el llenguatge (Sánchez i Díaz 2014; Domeniconi i Gràcia, 2018). Una gran part dels logopedes dels Centres de Desenvolupament infantil i Atenció Precoç comencen a replantejar-se el canvi i modificar les propostes d'intervenció (Dalmau et al. 2017). Des d'aquesta perspectiva, s'anima a presentar una pràctica centrada en la unitat familiar (més que en el nen); es reconeixen i es dona suport a les fortaleses i les competències de la família; es considera que és la família qui pot fomentar el desenvolupament i l'aprenentatge del llenguatge de manera més eficaç a través de les seves rutines (com per exemple en la lectura de contes). Es valoren totes aquelles activitats que impliquin la interacció positiva entre els adults i els infants, i que necessàriament es donen en els entorns naturals i inclusius del nen, per promoure el desenvolupament del llenguatge (Dunst, Raab, Trivette i Swanson 2010; McWilliam 2000, 2010). L'ECF anima els pares a participar de manera activa en els processos d'avaluació i identificació de les necessitats dels seus fills, la determinació dels objectius, els recursos, les adaptacions de l'entorn (físic, temporal i social), la tecnologia assistida i les estratègies que s'han d'incloure als plans d'intervenció per promoure l'accés i la participació dels nens en experiències que promoguin l'aprenentatge i el desenvolupament del llenguatge i la comunicació (DEC 2014; Meyer i Catalino 2016). En aquest sentit, els pares poden valorar com és el nivell de llenguatge dels seus fills a partir de valoracions comunicatives funcionals en el seu entorn natural i no només a partir de proves estandarditzades que proposin els logopedes i, a partir d'un treball en col-laboració, establir objectius comuns d'intervenció. El nou enfocament subratlla que la intervenció directa tradicional amb el nen hauria de ser substituïda i/o complementada amb un servei indirecte que donés orientació, formació i assessorament als agents educatius en l'entorn natural del nen aprofitant les situacions de les rutines diàries per intervenir en aquells aspectes de la parentalitat que promouen millors relacions comunicatives amb l'infant i com a conseqüència un millor desenvolupament del llenguatge oral (Epley, Summers i Turnbull 2010; Giné, Gràcia, Vilaseca i Balcells 2009; McWilliam 2016). Estudis basats en l'evidència, mostren que l'ECF millora les percepcions dels pares de les seves habilitats parentals (per exemple, la seva autoeficàcia en relació amb la criança); augmenta el benestar familiar contribuint a reduir la càrrega $i$ l'estrès que a vegades afecten les famílies; ajuda els pares a establir una millor relació amb els seus fills, i promou més eficaçment el desenvolupament infantil en àrees com el desenvolupament del llenguatge i la comunicació (Espe-Sherwindt i Serrano 2016).

\section{CONCLUSIONS}

El desenvolupament de la comunicació i del llenguatge és el resultat de la interacció de diversos factors (genètics, neurològics, interns i individuals, socials, culturals...). Allò exposat en el primer apartat de l'article ens permet concloure que les interaccions entre el bebè i els adults significatius durant el primer any de vida constitueixin les bases del desenvolupament comunicatiu i lingüístic posterior. Les capacitats dels nadons per interactuar amb les persones i processar la parla, el desenvolupament de les habilitats Parentalitat i parla adreçada als infants: com els adultrs promouen el desenvolupament 51 
d'atenció conjunta i el suport adult són aspectes fonamentals per entendre com se senten aquestes bases i la seva rellevància per al desenvolupament cognitiu i lingüístic posterior.

En aquest article hem posat l'èmfasi en el suport que els pares i les mares, i en general els adults significatius per als nens i les nenes, ofereixen als avenços comunicatius i lingüístics infantils en el context de les interaccions de la vida diària. D'acord amb els estudis presentats a l'apartat 2, podem concloure que l'afecte, la responsivitat, l'encoratjament o promoció de l'autonomia i l'ensenyança, en termes d'estimulació lingüística i cognitiva, que els adults proporcionen als infants en el marc de les activitats de la vida quotidiana, promouen el desenvolupament infantil en general i el desenvolupament comunicatiu i lingüístic en particular. L'apartat 3 ens mostra que les característiques pròpies de la parla adreçada a l’infant en el marc de la conversa són una peça clau del suport parental al procés de desenvolupament de la comunicació i del llenguatge.

Aprofundir en les característiques d'aquest suport i en el paper que aquest té en els processos de desenvolupament comunicatiu i lingüístic ens permet entendre millor com es donen aquests processos, així com fonamentar programes d'intervenció adreçats a optimitzar-los. El model centrat en la família i la intervenció naturalista i sociopragmàtica són, al nostre entendre, una via idònia per a la intervenció optimitzadora del desenvolupament comunicatiu i lingüístic, d'acord amb els resultats de les investigacions a les quals ens hem referit al llarg d'aquest article.

\section{REFERÈNCIES BIBLIOGRÀFIQUES}

Aslin, Richard N.; Pisoni, David B.; Jusczyk, Peter W. (1983). Auditory development and speech perception in infancy. Mussen, Paul H. (comps.). Handbook of child psychology (4 ${ }^{\text {a }}$ edició), Vol. 1, HaITH, Marshall; CAMPos, JoSePH J. (Comps.). Infant and developmental psychopathology. New York: Wiley.

BINGHAM, GARY E. (2007). Maternal Literacy Beliefs and the Quality of Mother-Child Book-reading Interactions: Associations with Children's Early Literacy Development. Early Educational Development, 18(1), 23-49.

Bosch, Laura; Sebastián-Gallés, Núria (2003). Simultaneous Bilingualism and the Perception of a Language-Specific Vowel Contrast in the First Year of Life. Language and Speech, 46(2-3), 217-243.

BRENT, MiChAEL R.; SISKIND, JEFFREY M. (2001). The role of exposure to isolated words in early vocabulary development. Cognition, 81(2), 33-44.

BRoEn, PATRICIA (1972). The verbal environment of the language-learning child. ASHA Monographs, 17.

Bruner, Jerome (1983). Childs talk. New York: Norton (Ed. cat.: La parla dels infants. Vic: Eumo, 1985).

Burnham, Denis; Kitamura, Christine; Vollmer-Conna, Uté (2002). What's new, pussycat? On talking to babies and animals. Science, 296(5572), 1435.

CAbrera, Natasha J.; Fagan, Jay; Wight, Vanessa; Schadler, Cornelia (2011). Influence of Mother, Father, and Child Risk on Parenting and Children's Cognitive and Social Behaviors. Child Development, 82(6), 1985-2005.

Carpenter, Malinda; Nagell, Katherine; Tomassello, Michael (1998). Social cognition, joint attention and communicative competence from 9 to 15 months of age. Monographs of the Society for Research in Child Development, 63(4), 1-143. 
Cielinski, Kerry L.; Vaughn, Brian E.; Seifer, Ronald; Contreras, Josefina (1995). Relations among sustained engagement during play, quality of play, and mother-child interaction in samples of children with Down syndrome and normally developing toddlers. Infant Behavior and Development, 18(2), 163-176.

Dalmau, Mariona; Balcells-Balcells, Anna; Giné, Climent; Cañadas, Margarita; Casas, Olga; Salat, Yolanda; Farré, Verònica; Calaf, Núria (2017). Como implementar el modelo centrado en la familia en Atención Precoz. Anales de Psicología, 33(3), 641-651.

Del Rio, MARía José (2006). Consideraciones sobre el uso de los procedimientos naturalistas para la intervención en logopedia. Revista de Logopedia, Foniatría y Audiología, 26(3), 139-145.

Division for Early Childhood (2014). DEC recommended practices in early intervention/early childhood special education 2014. Retrieved from: $<$ http://www.decsped.org/dec-recommended-practices > [Consulta: 4 març 2019].

DOMENICONI, CAMILA; GRÀcIA, MARTA (2018). Efectos de una intervención siguiendo el modelo enfocado en la familia para promover avances en el desarrollo del lenguaje de los niños. Revista de investigación en Logopedia, 8(2). 165-181.

Dunst, CARL J.; ESPE-Sherwindt, MARILYN (2016). Family-centered practices in early childhood intervention. REICHOW, BRIAN; BOYD, BRIAN; BARTON, ERIN; OdOM, SAMUEL (eds.). Handbook of Early Childhood Special Education (37-55). New York, NY: Springer.

Dunst, Carl J.; Raab, Melinda; Trivette, Carol M.; Swanson, Jennifer (2010). Community-based everyday child learning opportunities. MCWilliaM, RoBIN A. (ed.). Working with families of young children with special needs (60-92). New York, NY: Guilford Press.

Epley, Pamela; Summers, Jean; Turnbull, Ann (2010). Characteristics and trends in family-centered conceptualizations. Journal of Family Work, 13(3), 269-285.

ESPE-SHERWINDT, MARILYN; SERRANO, ANA MARIA (2016). It takes two: the role of familycentered practices in communication intervention. Revista de logopedia, Foniatría y Audiología, 36(4), 162-169.

GaRniCA, Olga (1977). Some prosodic and paralinguistic features of speech to Young children. Snow, Catherine E.; Ferguson, Charles A. (eds.). Talking to children: language input and acquisition (63-88). Cambridge: Cambridge University Press.

Giné, Climent; GrÀcia, Marta; Vilaseca, Rosa Maria; Balcells, AnNa (2009). Trabajar con las familias en atención temprana [Working with families in early intervention]. Revista Interuniversitaria de Formación del Profesorado,65(23) 95-113.

Girolametto, Luigi; Tannock, Rosemary (1994). Correlates of directiveness in the interactions of fathers and mothers of children with developmental delays. J. Speech Hear. Res., 37, 1178-1191.

Goldstein, Michael H.; Schwade, Jennifer A. (2008). Social feedback to infants' babbling facilitates rapid phonological learning. Psychological Science, 19(5), 515-523.

GRÀCIA, MARTA (2000). Intervención naturalista en la comunicación y el lenguaje para familias de niños pequeños con síndrome de Down. Revista Síndrome de Down, 17(1), 214 .

Hirsh-Pasek, Kathy; Kemler Nelson, Deborah G.; Jusczyk, Peter W.; Cassidy, KIMBERLY W.; DRUSS, BENJAMIN; KENNEDY, LORI (1987). Clauses are perceptual units for young infants. Cognition, 26(3), 269-286. 
HOFF, ERIKA (2006). How social contexts support and shape language development. Developmental Review, 26(1), 55-88. <http://doi.org/10.1016/j.dr.2005.11.002> [Consulta: 16 març 2019].

Hubbs-Tait, laura; Culp, Anne McDonald; Culp, Rex E.; Miller, Carrie E. (2002). Relation of maternal cognitive stimulation, emotional support, and intrusive behavior during Head Start to children's kindergarten cognitive abilities. Child Development, 73(1), 110-131.

Kaye, Kenneth (1982). The mental and social life of babies: How parents create persons. London: Harvester Press (ed. cast.: La vida mental y social del bebé. Cómo los padres crean personas. Barcelona: Paidós, 1986).

Kim-Cohen, Julia; Moffitt, Terrie E.; Caspi, Avshalom; Taylor, Alan (2004). Genetic and environmental processes in young children's resilience and vulnerability to socioeconomic deprivation. Child Development, 75(3), 651-668.

KOCHANSKA, GRAZYNA (2001). Emotional development in children with different attachment histories: The first three years. Child Development, 72(2), 474-490.

KochansKa, Grazyna; Kim, SANGHAG (2013). Difficult temperament moderates links between maternal responsiveness and children's compliance and behavior problems in low-income families. Journal of Child Psychology and Psychiatry and Allied Disciplines, 54(3), 323-332. <http://doi.org/10.1111/jcpp.12002> [Consulta: 20 març 2019].

LAmb, Michael E. (ed.). (2010). The role of the father in child development. Hoboken, NJ: John Wiley.

LEUNGH, ElEANOR H.; RHEINGOLD, HARRIET L. (1981). Development of pointing as a social gesture. Developmental Psychology, 17(2), 215-220.

LiU, H. M.; Kuhl, P. K.; TsaO, F. M. (2003). An association between mothers' speech clarity and infants' speech discrimination skills. Developmental Science, 6(3), 1-10. <http://doi.org/10.1111/1467-7687.00275> [Consulta: 22 març 2019].

McGillion, Michael; Pine, Julian; Herbert, Jane; Matthews, Danielle (2017). A randomised controlled trial to test the effect of promoting caregiver contingent talk on language development in infants from diverse socioeconomic status backgrounds. Journal of Child Psychology and Psychiatry, 58(10), 1122-1131.

McRoberts, Gerald W.; McDonough, Colleen; Lakusta, Laura (2009). The role of verbal repetition in the development of infant speech preferences from 4 to 14 months of age. Infancy, 14(2), 162-94.

McWilliam, Robin A. (2000). It's only natural... to have early intervention in the environments where it's needed. SANDAll, Susan REBecka; Ostrosky, Michaelene (eds.). Young Exceptional Children Monograph Series No. 2: Natural Environments and Inclusion (pp. 17-26). Denver, CO: The Division for Early Childhood of the Council for Exceptional Children.

-- (2010). Routines-based early intervention. Supporting Young Children and Their Families. Baltimore, MD: Paul H. Brookes.

-- (2016). Birth to three early intervention. Reichow, B.; Boyd, B.; Barton, E.; Odom, S. (eds.). Handbook of Early Childhood Special Education (pp. 75-88). New York, NY: Springer.

Mehler, JaCQues; Betoncini, Josiane; BARrière, Michele; JASSIK-Gerchenfeld, DorA (1978). Infant recognition of mother's voice. Perception, 7(5), 491-497.

Meltzoff, ANdrew N.; Moore, M. Keith (1997). Explaining facial imitation: A theoretical model. Early Development and Parenting, 6(3-4), 179-192. 
Meuwissen, Alyssa S.; CARLSON, STEPHANie M. (2015). Fathers matter: The role of father parenting in preschoolers' executive function development. Journal of Experimental Child Psychology, 140, 1-15.

Meyer, Lori; CATAlino, Tricia (2016). Environments that promote young children's participation. Implementing the DEC recommended practices. Retrieved from $<$ https://illinois.edu/blog/files/6039/140057/83588.pdf> [Consulta: 2 març 2019].

Moon, Christine; CoOper, Robin P.; Fifer, William P. (1993). Two-day-olds prefer their native language. Infant Behavior and Development, 16(4), 495-500.

Murray, LynNe; TREVARThen, COLWYN (1985). Emotional regulation of interactions between two-montholds and their mothers. Field, T. M.; Fox, N. A. (eds.). Social Perception in Infants (pp. 177- 197). Norwood, NJ: Ablex Publishers.

Murray, Lynne; Trevarthen, Colwyn (1986). The infant's role in mother-infant communications. Journal of Child Language, 13(1), 15-29.

Newman, Rochelle S.; Rowe, Meredith L.; Bernstein Ratner, Nan (2015). Input and uptake at 7 months predicts toddler vocabulary: the role of child-directed speech and infant processing skills in language development. Journal of Child Language, 43(5), 1-16. <http://doi.org/10.1017/S0305000915000446> [Consulta: 19 març 2019].

Nicely, Pamela; Tamis-LeMonda, CATHerine S.; Bornstein, Marc H. (1999). Mothers' attuned responses to infant affect expressivity promote earlier achievement of language milestones. Infant Behavior and Development, 22(4), 557-568.

Ninio, ANAT (1993). On the fringes of the system: Children's acquisition of syntactically isolated forms at the onset of speech. First Language, 13, 291-313.

OchS, Elinor; SchiEfFElin, BAMBi (1995). The impact of language socialization on gramatical development. FleTcher, PAUl; MACWhinNEY, Brian (eds.). The Handbook of Child Language (pp. 73-94). Oxford: Blackwell.

Pan, B. A.; Imbens-Bailey, A.; Winner, K.; Snow, C. (1996). Communicative intents expressed by parents in interaction with young children. Merrill-Palmer Quarterly, $42(2), 248-266$.

Piaget, JeAn (1936). La naissance de lintelligence chez l'enfant. Neuchâtel i Paris: Delachaux et Niestlé (Ed. cast.: Los orígenes de la inteligencia en el niño. Madrid: Aguilar, 1969).

Prieto, P.; Esteve-Gibert, N.; Igualada, A.; Bosch, L.; Pons, F.; Solé, J. (2015). Abans de les primeres paraules: Recercaixa. <http://abansprimeresparaules.upf.edu $>$ [Consulta: 20 març 2019].

Rivero, Magda (1993). La influencia del habla de estilo materno en la adquisición del lenguaje: valor y límites de la hipótesis del input. Anuario de Psicología, 57(2), 45-64.

-- (2006). Aprendre a expressar intencions comunicatives a través del llenguatge. Comunicació Educativa, 19, 49-55. <https://doi.org/10.17345/comeduc200649-55> [Consulta: 4 abril 2019].

-- (2010). Maternal expression of communicative intentions and pragmatic fine tuning in early infancy. Infant Behavior \& Development, 33(4), 373-86.

-- (2018). Los inicios de la comunicación. En V. Torrens (Coord.). La adquisición del lenguaje (p. 9-56). London: Pearson.

Rivero, Magda; Fernández-Viader, Maria Del Pilar; Gràcia, Marta (1995). Evolución de los turnos de conversación en la interacción madre-hijo. Revista de Logopedia, Foniatría y Audiología, XV(3), 164-173. 
Rivero, MAgda; Vilaseca, Rosa; Borneis, SANDRA (juliol de 2017). The role of parenting and Child Directed Speech in child language development. Pòster presentat al 14th International Congress for the Study of Child Language, Lyon, França.

Rivero, Magda; Vilaseca, Rosa; Bersabé, Rosa; Navarro-Pardo, EsPeranza; Cantero, María José; Ferrer, Fina; VAlls, Clara (març de 2019). Interacciones parentales positives de madres y padres durante el juego con sus hijos pequeños en el hogar. Pòster presentat al I Congreso Internacional de Educación e Intervención: Psicoeducativa, Familiar y Social, Las Palmas de Gran Canaria, Espanya.

Roggman, Lori A.; Cook, Gina A.; Innocenti, Mark S.; Jump Norman, Vonda K.; Christiansen, KATIE (2013b). PICCOLO, Parenting Interactions with Children: Checklist of Observations linked to Outcomes. Logan, UT: Utah State University.

Rowe, MEREDiTh L. (2012). A longitudinal investigation of the role of quantity and quality of child-directed speech vocabulary development. Child Development, 83(5), 1762-1774. <http://doi.org/10.1111/j.1467-8624.2012.01805.x> [Consulta: 4 abril 2019].

SACHS, J. (1977). The adaptative significance of linguistic input to prelinguistic infants. Snow, Catherine E.; Ferguson, Charles (eds.). Talking to children: language input and acquisition (pp. 51-61). Cambridge: Cambridge University Press.

SÁnCHEZ LóPEZ, Amparo; DíAZ SÁnChEZ, CRistina (2014). Experiencias desde el ámbito de la logopedia y proceso de transformación de los CDIATS de Asprona. Actas del XXIX Congreso AELFA 2014: Logopedia: Evolución, Transformación y futuro. Murcia.

SÁnChEZ-CANo, MANuel (2001). Aprendiendo a hablar con ayuda. Lleida: Milenio

Serra, Miquel; Serrat, Elisabet; Solé, Maria Rosa; Bel, Aurora; Aparici, Melina (2000). La adquisición del lenguaje. Barcelona: Ariel.

Slater, Alan; Quinn, Paul C. (2001). Face recognition in the newborn infant. Infant and Child Development, 10(1), 21-24.

Snow, CAtherine E. (1977). Development of conversation between mothers and babies. Journal of Child Language, 4(1), 1-22.

-- (1995). Issues in the study of input: Fine-tuning, universality, individual and developmental differences, and necessary causes. Fletcher, P.; MacWhinney (eds.). The Handbook of Child Language (pp. 180-193). Oxford: Blackwell.

Snow, Catherine E.; Pan, Barbara A.; Imbens-Bailey, Alison; Herman, Jane (1996). Learning how to say what one means: a longitudinal study of children's speech act use. Social Development, 5(1), 56-84.

Tamis-LeMonda, Catherine S.; Bornstein, Marc H.; Baumwell, Lisa (2001). Maternal responsiveness and children's achievement of language milestones. Child Development, 72(3), 748-768.

Tamis-LeMonda, Catherine S.; Kuchirko, Yana; Song, Lulu (2014). Why Is Infant Language Learning Facilitated by Parental Responsiveness? Current Directions in Psychological Science, 23(2), 121-126.

Tomasello, Michael ( (1999). The cultural origins of human cognition. Cambridge, Massachusetts: Harvard University Press.

TOMASElLO, Michael; FARRAR, JefFrey (1986). Joint attention and early language. Child Development, 57, 1454-1463.

Trevarthen, Colwyn (1980). The foundations of intersubjectivity: Development of interpersonal and cooperative understanding in infants. OLsON, D. R. (eds.). The Social Foundations of Language and Thought (pp. 316-342). New York: Norton \& Company. 
-- (2015). Infant semiosis: The psycho-biology of action and shared experience from birth. Cognitive Development, 36, 130-141.

Tronick, EdWARd; Als, Heidelise; AdAMSOn, LaUREN (1979). Structure of early face-toface communicative interactions. BULlOwA, MARGARET (ed.). Before speech. The beginning of interpersonal communication. Cambridge: Cambridge University Press.

URQUía, BEGOÑa (1998). Intervención naturalista en contexto de aula en una escuela de educación especial. Revista de Logopedia, Foniatría y Audiología, 18(1), 31-41.

VILASECA, ROSA (2002). La intervención logopédica en niños con dificultades en el área del lenguaje y de la comunicación: Un enfoque naturalista. Revista de Logopedia, Foniatría y Audiología, 22(3), 143-150.

Vilaseca, Rosa; Rivero, Magda; Bersabé, Rosa Maria; Navarro-Pardo, Esperanza; Cantero, Maria Jose; Ferrer, Fina; Valls-Vidal, Clara; InNocenti, Mark S.; ROGGMAN, LORI (2019). Spanish Validation of the PICCOLO (Parenting Interactions With Children: Checklist of Observations Linked to Outcomes). Frontiers in Psychology. 10:680. doi: 10.3389/fpsyg.2019.00680.

Vilaseca, Rosa; Rivero, Magda; Ferrer, Fina; Cantero, Maria Jose; Bersabé, Rosa

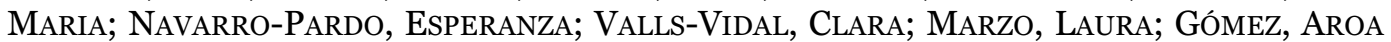
(abril-maig de 2018). Parentalidad positiva y desarrollo infantil en niños y niñas con discapacidad. Comunicació presentada al III Congreso Internacional sobre familias y discapacidad, Universitat Ramon Llull, Barcelona, Espanya.

Vouloumanos, AThena; Onishi, KRistine H.; Pogue, AmandA (2012). Twelve-month-old infants recognize that speech can communicate unobservable intentions. Proceedings of the National Academy of Sciences of the United States of America, 109(32), 12933-12937.

Vouloumanos, Athena; Werker, Janet F. (2007). Listening to language at birth: evidence for a bias for speech in neonates. Developmental Science, 1O(2), 159-164.

Zhou, Qing; Eisenberg, Nancy; Losoya, Sandra; Fabes, Richard A.; Reiser, Mark; GuTHRIE, IVANNA K. et al. (2002). The relations of parental warmth and positive expressiveness to children's empathy-related responding and social functioning: A longi 